\title{
Optimal Selective Transmission under Energy Constraints in Sensor Networks
}

\author{
Rocío Arroyo-Valles, Student Member, IEEE, Antonio G. Marques, Member, IEEE \\ and Jesús Cid-Sueiro, Senior Member, IEEE
}

\begin{abstract}
An optimum selective transmission scheme for energylimited sensor networks where sensors send or forward messages of different importance (priority) is developed. Considering the energy costs, the available battery, the message importances and their statistical distribution, sensors decide whether to transmit or discard a message so that the importance sum of the effectively transmitted messages is maximized. It turns out that the optimal decision is made comparing the message importance with a time-variant threshold. Moreover, the gain of the selective transmission scheme, compared to a non-selective one, critically depends on the energy expenses, among other factors. Albeit suboptimal, practical schemes that operate under less demanding conditions than those for the optimal one are developed. Effort is placed into three directions: (i) the analysis of the optimal transmission policy for several stationary importance distributions; (ii) the design of a transmission policy with invariant threshold that entails asymptotic optimality; and (iii) the design of an adaptive algorithm that estimates the importance distribution from the actual received (or sensed) messages. Numerical results corroborating our theoretical claims and quantifying the gains of implementing the selective scheme close this paper.
\end{abstract}

Index Terms-sensor networks, wireless sensor networks, energyaware systems, stochastic programming, message sending

\section{1 introduction - Problem Motivation}

$\mathrm{D}$ URING the last years, Sensor Networks have attracted the attention of many researchers from electronics, signal processing, communications, and networking communities [1]. The ability of sensors to behave in an autonomous and selforganized manner using limited energy and computation resources [2] states new challenges that require novel solutions.

In many practical scenarios, sensor node batteries cannot be (easily) refilled and nodes have a finite lifetime. Since communication processes are energy-expensive, the cost of transmitting and receiving information should influence node decisions; see, e.g., [3] or [4]. Typically, nodes are compelled to transmit any signal captured by their sensors while batteries are alive. A similar situation occurs in scenarios where nodes act as relays that have to forward any message upon request from other neighboring node. This inability to apply autonomous transmission policies, thus preventing nodes from

R. Arroyo-Valles and J. Cid-Sueiro are with the Dept. of Teoría de la Señal y Comunicaciones, Universidad Carlos III de Madrid, Avda. de la Universidad 30, Leganés, 28919, Madrid, Spain.E-mails: (marrval@tsc.uc3m.es, jcid@tsc.uc3m.es).

Antonio G. Marqués is with the Dept. of Teoría de la Señal y Comunicaciones, Universidad Rey Juan Carlos de Madrid, Camino del Molino s/n, Fuenlabrada 28943, Madrid, Spain.E-mail: (antonio.garcia.marques@urjc.es) managing their own resources, hinders an efficient utilization of the network. Some strategies to extend wireless sensor networks lifetime were early proposed; e.g., by establishing inactivity periods in node operation lifetime [5] or by adopting distributed digital signal processing (DDSP) techniques to reduce the amount of transmitted data [6].

Resource-saving strategies based on the nature of the information to be transmitted and the expected available resources in the sensors that take part in communication at each moment, have barely been analyzed in the literature. Nevertheless, there exist many practical scenarios where it is feasible to attribute a particular significance [7]; priority [8]; relevance [9]; or utility [10] value to messages transmitted or forwarded by sensor nodes. Tailored to those scenarios, we will consider that messages can be valuated through an importance indicator which reflects the priority of the message, the relevance of the information conveyed or the required level of quality of service (QoS). Relevant examples in the context of Sensor Networks can be found in the fields of: security (attack reports [7]), medical care (critical alerts [11]), or data fusion (DAIDA algorithm in [12]), to name a few.

Summarizing, in this paper we address the design of efficient transmission policies for Sensor Networks, constraining ourselves to applications where: (i) message importance can be properly quantified, and (ii) low graded messages can be eventually discarded. This way, we propose selective transmitters that save energy by discarding low priority messages with the expectation of sending more important upcoming messages.

Related ideas have recently been explored in literature. The IDEALS algorithm [13], built under the concept of message and power priorities, tries to extend network lifetime for important messages, discarding all messages except those of high importance when battery resources are scarce. The PGR (Prioritized Geographical Routing) algorithm [14] selects the appropriated routing technique depending on the priority of the message (low, medium or high). Moreover, a fuzzy logic approach to deal with message transfer priority arbitration that considers fifteen different priority levels has been presented in [15]. Nevertheless, none of these algorithms have been proposed under a probabilistic and statistical approach that may open the door to a long-term optimization of the network. A selective forwarding scheme using a probabilistic approach has recently been explored in [16], [17] and [18]. In those works, the decision to transmit or discard a message at each node is 
taken in order to minimize a cost which depends on the energy expenses as well as the message importance. The resulting protocols promote the transmission and forwarding of highly graded messages and, as a result, the overall importance of the transmitted messages during the full node lifetime is higher than the corresponding to non-selective methods. However, the relative influence of energy expenses and importance values in the overall cost is a heuristic parameter in the model, whose value is difficult to optimize.

Different from the aforementioned works, in this paper we develop an optimum selective message forwarding scheme based on a statistical model of the message importances. More specifically, we derive optimal decisions maximizing the importance sum of the transmitted messages at each node, and analyze the node behavior under different importance distributions. Using asymptotic analysis, gains with regard to a nonselective scheme are theoretically quantified. Furthermore, in scenarios where nodes do not know the statistical importance distribution of messages, an alternative method that does not require a priori knowledge of the statistical information is developed.

Noticeably, the statistical model here presented exhibits similarities to other problems in Operations Research and Stochastic Dynamic Programming (see, e.g., [21]), and the equations describing the energy evolution at the sensor node and the importance sum can be restated as a particular type of Markov decision process. Nonetheless, our treatment of the problem and the theoretical derivations are self-contained.

Finally, remark that the sensor model in this paper is an abstraction of reality which makes some simplifying assumptions: perfect transmissions, perfect knowledge of energy resources and importance distributions, and some others. Some of these assumptions are likely not critical, and the model can be modified in order to incorporate more realistic situations. Some others state some challenges in order to obtain more practical decision schemes. In any case, we believe that our model captures the essential behavior of a selective transmission scheme, and can be used as a starting point for other designs more accurately adapted to specific scenarios.

The rest of the paper is organized as follows. Section 2 describes the context and the sensor model. Section 3 focuses on the optimal selective transmitter, obtaining a general formula to compute the optimal time-variant threshold, which is thereafter particularized for specific operating conditions. Section 4 describes a selective transmission policy based on a constant threshold. The asymptotic analysis in Section 5 provides a gain formula as well as some illustrative examples. In Section 6, an adaptive method which estimates the importance distribution on-the-fly is presented. Section 7 is in charge of showing the experimental study and results for a single node and a sensor network. Some conclusions and pointers to future work close the paper in Section 8.

\section{Sensor MOdel}

For the purpose of the analysis that follows, we consider a sensor network as a collection of nodes $\mathcal{N}=\{n \mid n=$ $0, \ldots, N-1\}$. For the time being, we will focus on the behavior of each node, which receives a sequence of requests to transmit messages (no matter how the network topology is). The node dynamics will be characterized by two variables

- $e_{k}$ : available energy at a given node at time $k$. It reflects the "internal state" of the node; and

- $x_{k}$ : importance of the message to be sent at time $k$. It reflects the "external input" to the node.

For mathematical reasons, we assume that if the node does not receive any request to transmit at time $k$, then $x_{k}=0$, while true messages will have $x_{k}>0$.

At time $k$, the sensor node must make a decision, $d_{k}$, about sending or not the current message, so that $d_{k}=1$ if the message is sent, and $d_{k}=0$ if the node decides to discard it.

Nodes consume energy at each time slot, by an amount that depends on the message reception and the taken actions. In the literature, up to three different energy expenses are typically considered:

- $E_{I}$ : energy spent at a silent time, when there is no message reception, and the node may stay at "idle" mode;

- $E_{R}$ : energy spent when receiving a message; and

- $E_{T}$ : energy spent when transmitting a message.

The value of these parameters will depend on the system specifications and the specific application (among the factors that will determine the energy costs we find mobility, sensed magnitude, or behavior of the batteries, to name a few). For example, for static dense networks, $E_{T}$ and $E_{R}$ values may be very similar, while for mobile networks operating over fading channels, $E_{T}>>E_{R}$ is expected.

Energy at time $k$ can be expressed recursively as

$$
e_{k+1}=e_{k}-d_{k} E_{1}\left(x_{k}\right)-\left(1-d_{k}\right) E_{0}\left(x_{k}\right),
$$

where $E_{1}\left(x_{k}\right)$ is the energy consumed when the node decides to transmit the message, and $E_{0}\left(x_{k}\right)$ is the energy consumed when the message is discarded. For positive values of importance, energy consumption is independent of the message importance, and we have

$$
\begin{aligned}
& E_{1}\left(x_{k}\right)=E_{T}+E_{R}, \quad x_{k}>0 \\
& E_{0}\left(x_{k}\right)=E_{R}, \quad x_{k}>0 .
\end{aligned}
$$

Recalling that $x_{k}=0$ means that no messages are received, we also have

$$
E_{1}(0)=E_{0}(0)=E_{I}
$$

When the sensor node is the source of the message, $E_{R}$ comprises the energy expense of the message generation process (possibly by a sensing device). When the sensor node acts as a forwarder, $E_{R}$ comprises the energy expense of receiving the message from other node. Thus, we assume that $E_{R}$ is the same no matter if the node is the source of the message or it has been requested to forward a message from other node. Even though this assumption is not critical and could be bypassed by splitting $E_{R}$ between receiving and sensing costs, we adopt it for two reasons: (i) it leads to a simpler mathematical formulation and (ii) nodes are prevented from acting selfishly (note that if the energy cost of sensing were smaller than the cost of receiving, nodes would promote their own messages instead of forwarding others' messages). 
Remark 1: It is important to mention that although this paper focuses on the case where the energy consumption is given by (2)-(4), we will formulate and solve the general case in (1) by assuming that both consumption profiles, $E_{1}(x)$ and $E_{0}(x)$, may arbitrarily depend on $x$. As a first approach, the model could even be applied to situations where $E_{T}$ and $E_{R}$ are random or time-variant (e.g., in sensors operating over fast fading channels where transmissions are adapted based on the channel state information) by substituting $E_{T}$ and $E_{R}$ by their respective mathematical expectations. In any case, we assume that both energy functions are perfectly known.

\section{Optimal Selective tRansmission}

To derive the optimal transmission policy we will consider that node decisions do not depend on the state and the actions of neighboring nodes, but only on the available information at each node. Therefore, at each time, $k$, the node decision depends on the internal state and the external input

$$
d_{k}=g\left(e_{k}, x_{k}\right)
$$

with the constraint

$$
g\left(e_{k}, x_{k}\right)=0, \text { if } e_{k}<E_{1}\left(x_{k}\right)
$$

reflecting that, if the sensor node does not have enough energy to receive and transmit the message, it cannot decide $d_{k}=1$.

Decisions at each node will be made with infinite horizon, i.e., by maximizing (on average) the importance sum of all transmitted messages ${ }^{1}$

$$
s_{\infty}=\sum_{k=0}^{\infty} d_{k} x_{k}
$$

Since nodes have limited energy resources, this sum only contains a finite number of nonzero values (eventually, for some $k, e_{k}<\min _{k} E_{1}\left(x_{k}\right)$, and $\forall k^{\prime} \geq k$, we have $d_{k^{\prime}}=0$ ).

The following result provides the optimal selective transmitter.

Theorem 1: Let $\left\{x_{k}, k \geq 0\right\}$ be a statistically independent sequence of importance values, and $e_{k}$ the energy budget at time $k$, whose energy process is given by (1). The optimal sequence of decision rules in the form $d_{k}=g\left(e_{k}, x_{k}\right)$ maximizing $\mathbb{E}\left\{s_{\infty}\right\}$ (with $g\left(e_{k}, x_{k}\right)=0$ for $e_{k}<E_{1}\left(x_{k}\right)$ and $s_{\infty}$ given by (7)), is

$$
d_{k}=u\left(x_{k}-\mu_{k}\left(e_{k}, x_{k}\right)\right) u\left(e_{k}-E_{1}\left(x_{k}\right)\right),
$$

where $u(x)$ stands for the Heaviside step function (with the convention $u(0)=1$ ) and thresholds $\mu_{k}$ can be computed recursively through the pair of equations

$$
\mu_{k}\left(e_{k}, x_{k}\right)=\lambda_{k+1}\left(e_{k}-E_{0}\left(x_{k}\right)\right)-\lambda_{k+1}\left(e_{k}-E_{1}\left(x_{k}\right)\right)
$$

1. Since our design focuses on the performance of each single node, decisions made at other nodes are not explicitly taken into account, but only implicitly through messages actually received from its neighbors. Although this approach fits into the design philosophy of sensor networks where the complexity of each node should be kept as low as possible, it is worth remarking that from an overall network perspective, it may entail a loss of performance. Interestingly, preliminary results published in [19] show that the loss of optimality due to this simplified design is very small, therefore validating the proposed approach.

$$
\begin{aligned}
\lambda_{k}\left(e_{k}\right) & =\left(\mathbb{E}\left\{\lambda_{k+1}\left(e_{k}-E_{0}\left(x_{k}\right)\right)\right\}\right. \\
& \left.+\mathbb{E}\left\{\left(x_{k}-\mu_{k}\left(e_{k}, x_{k}\right)\right)^{+} u\left(e_{k}-E_{1}\left(x_{k}\right)\right)\right\}\right) u\left(e_{k}\right),
\end{aligned}
$$

where

$\left(x_{k}-\mu_{k}\left(e_{k}, x_{k}\right)\right)^{+}=\left(x_{k}-\mu_{k}\left(e_{k}, x_{k}\right)\right) u\left(x_{k}-\mu_{k}\left(e_{k}, x_{k}\right)\right)$.

The auxiliary function $\lambda_{k}(e)$ represents the expected increment of the total importance (expected reward) at time $k$, i.e.,

$$
\lambda_{k}(e)=\sum_{i=k}^{\infty} \mathbb{E}\left\{d_{i} x_{i} \mid e_{k}=e\right\} .
$$

Proof: See Appendix A.

Although the results of Theorem 1 are general and hold for any energy cost and importance value, they do not provide a clear intuition about the impact of $E(x)$ and the distribution of $x_{k}$ on the design of the optimal forwarding scheme. Moreover, the direct application of these results is difficult, because (9) and (10) state a time-reversed recursive relation: in order to make optimal decisions, the node should know the future importance distributions in advance. For these reasons, in the reminder of this paper we will focus special attention on several particular cases that will lead us to tractable closedform solutions.

\subsection{Stationarity}

If all variables $x_{1}, \ldots, x_{k}$ have the same distribution, then $\mu_{k}$ does not depend on $k$ [c.f. (9) and (10)]. In this case, the following result can be shown:

Theorem 2: Under the conditions of Th. 1, if the importance values $\left\{x_{k}, k \geq 0\right\}$ are identically distributed and $\inf _{x}\left\{E_{i}(x)\right\}>0$, for $i=0,1$, the optimal sequence of decision rules in the form $d_{k}=g\left(e_{k}, x_{k}\right)$ maximizing $\mathbb{E}\left\{s_{\infty}\right\}$ (with $g\left(e_{k}, x_{k}\right)=0$ for $e_{k}<E_{1}\left(x_{k}\right)$ and $s_{\infty}$ given by (7)), is

$$
d_{k}=u\left(x_{k}-\mu\left(e_{k}, x_{k}\right)\right) u\left(e-E_{1}\left(x_{k}\right)\right),
$$

where

$$
\begin{aligned}
& \mu(e, x)=\lambda\left(e-E_{0}(x)\right)-\lambda\left(e-E_{1}(x)\right) \\
\lambda(e)= & \left(\mathbb{E}\left\{\lambda\left(e-E_{0}(x)\right)\right\}\right. \\
+ & \left.\mathbb{E}\left\{(x-\mu(e, x))^{+} u\left(e-E_{1}(x)\right)\right\}\right) u(e),
\end{aligned}
$$

Proof: See Appendix B.

It is important to stress that in most scenarios involving multiple sensors, the stationarity assumption, strictly speaking, is not true. For example, the distribution of messages arriving to a node depends on the transmission policy used by forwarding nodes. Since the optimal policy presented here is energy-dependent [c.f. either (9) or (14)] and the available energy clearly changes along time for all nodes, the importance distribution of the received messages will also change along time. However, it will be shown in the next sections that the simplification obtained in (14) is not only useful from a theoretical perspective, but also valid from a practical point of view for large networks. This (almost) stationary behavior can be justified based on different reasons. First, although 
the optimal forwarding policy varies along time, this variation turns out to be negligible during most of the time (i.e., it is almost-stationary). The underlying reason is that for mediumhigh values of available energy the optimal forwarding scheme is not very sensitive to energy changes. Only when nodes are close to run out of batteries, the decision threshold varies significantly as a function of the remaining energy. Second, even if the behavior of a single node is not stationary, the aggregate effect of the entire network may be stationary. In other words, the approximation given by (14) will be accurate during most of the time, and the discrepancy will only arise when the network is close to expire. Theoretical analysis and numerical results will corroborate this intuition.

\subsection{Constant energy profiles}

Under the constant profile model given by (2)-(4), the optimal threshold can be written as

$$
\mu_{k}(e, x)=\mu_{k}(e) I_{x>0}
$$

where $I_{x>0}$ is an indicator function (equal to unity if the condition holds and zero otherwise), and using (9) we have

$$
\mu_{k}(e)=\lambda_{k+1}\left(e-E_{R}\right)-\lambda_{k+1}\left(e-E_{T}-E_{R}\right) .
$$

Also, (10) becomes

$$
\begin{aligned}
\lambda_{k}(e)= & P_{I} \lambda_{k+1}\left(e-E_{I}\right)+\left(1-P_{I}\right) \lambda_{k+1}\left(e-E_{R}\right) \\
& -P_{I} \mu_{k}(e, 0) u\left(-\mu_{k}(e, 0)\right) u\left(e-E_{I}\right) \\
& +\left(1-P_{I}\right) \mathbb{E}\left\{\left(x_{k}-\mu_{k}\left(e, x_{k}\right)\right)^{+} \mid x_{k}>0\right\} \\
& \cdot u\left(e-E_{T}-E_{R}\right) \\
= & P_{I} \lambda_{k+1}\left(e-E_{I}\right)+\left(1-P_{I}\right) \lambda_{k+1}\left(e-E_{R}\right) \\
& +\left(1-P_{I}\right) \mathbb{E}\left\{\left(x_{k}-\mu_{k}\left(e, x_{k}\right)\right)^{+} \mid x_{k}>0\right\} \\
& \cdot u\left(e-E_{T}-E_{R}\right)
\end{aligned}
$$

where $P_{I}=\operatorname{Pr}\{x=0\}$. Defining

$$
H_{k}(\mu)=\mathbb{E}\left\{\left(x_{k}-\mu\right)^{+} \mid x_{k}>0\right\},
$$

we can write

$$
\begin{aligned}
\lambda_{k}(e) & =P_{I} \lambda_{k+1}\left(e-E_{I}\right)+\left(1-P_{I}\right) \lambda_{k+1}\left(e-E_{R}\right)+ \\
& +\left(1-P_{I}\right) H\left(\mu_{k}(e)\right) u\left(e-E_{T}-E_{R}\right) .
\end{aligned}
$$

Thus, the optimal transmission policy for a sensor with a constant energy profile is described by (17) and (20). In order to analyze the influence of idle times and the relation between transmission and reception energy expenses separately, in the following examples we consider the case of $P_{I}=0$ and/or $E_{I}=0$. Note that if any of these conditions holds, the expected importance sum in (20) can be rewritten as

$$
\lambda_{k}(e)=\lambda_{k+1}\left(e-E_{R}\right)+H\left(\mu_{k}(e)\right) u\left(e-E_{T}-E_{R}\right) .
$$

\subsection{Examples}

As we have already mentioned, there is no general explicit solution to the pair of equations (9) and (10), not even for the stationary case in (17) and (20). For this reason, in this section we focus on systems satisfying the operating conditions that gave rise to (21) (constant energy profiles, stationarity and zero idle energy) and solve the recursive relations for several importance distributions ${ }^{2}$. This simplification will lead to tractable expressions, providing insight into the behavior of the optimal forwarding scheme.

- Uniform Distribution: Let $U(0,2)$ denote the uniform distribution between 0 and 2 whose probability density function (PDF) is

$$
p(x)=\frac{1}{2}(u(x)-u(x-2)) .
$$

Substituting (22) into (19), we have

$$
H(\mu)=E\left\{(x-\mu)^{+}\right\}=\frac{1}{4}(2-\mu)^{2},
$$

and therefore, the expected reward is given by

$$
\lambda(e)=\lambda\left(e-E_{R}\right)+\frac{1}{4}(2-\mu(e))^{2} u\left(e-E_{T}-E_{R}\right) .
$$

Fig. 1(a) plots the threshold for extremely small values of available energy, e. $E_{1}(x)=1$ and different values of the ratio $E_{T} / E_{R}$ are considered. Note that, for values of $e$ lower than 1 , in spite of the threshold value is 0 , there is no actual transmission because $u\left(e-E_{T}-E_{R}\right)=0$. For $1<e<E_{1}+E_{R}$ there is only one opportunity to send the message, so the threshold is also 0 , which means that the message will be transmitted whatever its importance value is. For larger energy values, the threshold increases, meaning that the transmission can be made more selective. Note, also, that $\mu(e)$ evolves in a staircase manner, because any energy amount in excess of a multiple of $E_{R}$ is useless.

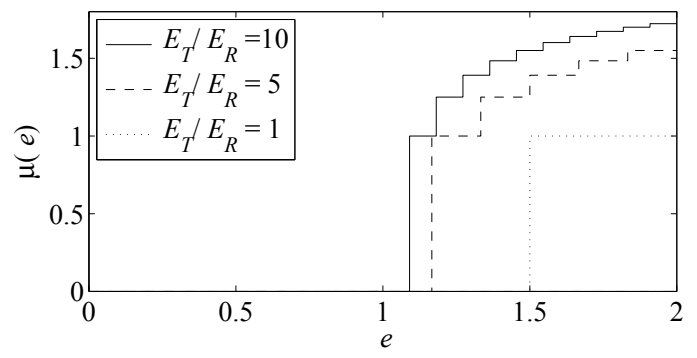

(a)

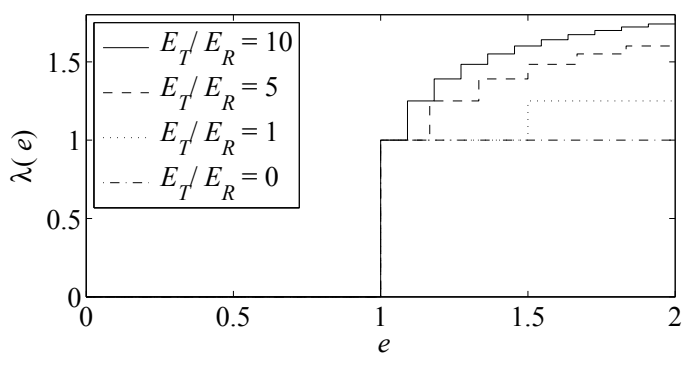

(b)

Fig. 1: Variation of the decision threshold (a) and the expected importance sum (b) with respect to the available energy, e. A uniform importance distribution $U(0,2)$ with $E_{1}(x)=E_{R}+E_{T}=1$ is assumed. Different plots correspond to different values of $E_{T} / E_{R}$.

2. In the following, free parameters will be set so that all importance distributions have a mean value equal to one. 
Fig. 1(b) represents the expected reward $(\lambda(e))$. Note that the case $E_{T}=0$ is equivalent to a non-selective transmitter (because, according to (17), the optimal threshold is 0 in that case, which means that no messages are discarded). Despite that, for $e$ close to 2, there is not energy for a second transmission, the selective transmitter provides a significant expected income with respect to the non-selective one.

Fig. 2(a) shows the optimal threshold for $E_{T}=4, E_{R}=$ 1 and high values of available energy. Note the sawtooth shape of the forwarding threshold: as the available energy is reduced to a value close to a multiple of the energy required to transmit, the forwarding threshold decreases, because if there is not any transmissions, the total number of possible messages to be sent is reduced by a unity.

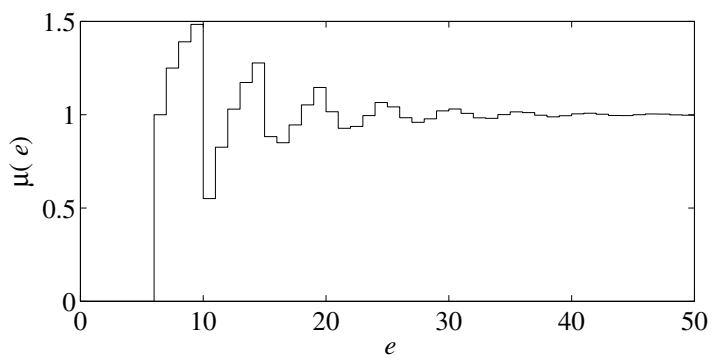

(a)

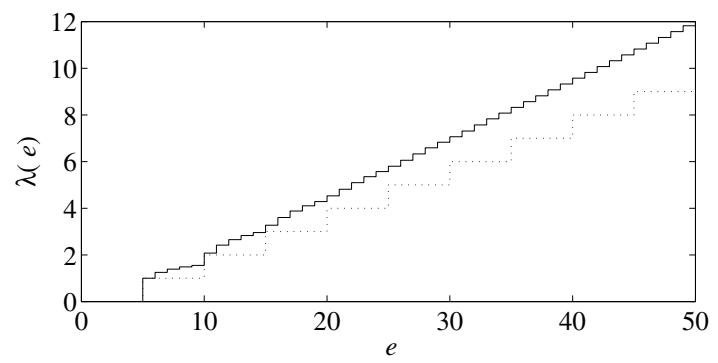

(b)

Fig. 2: The decision threshold (a) and the expected importance sum (b) (continuous line) as a function of the available energy. A uniform importance distribution $U(0,2)$ with $E_{T}=4$ and $E_{R}=1$ is assumed. The stepwise function (dotted line) reflects the behavior of a non-selective transmitter, which transmits any message whatever its importance value is.

Fig. 2(b) represents the expected reward of the selective transmitter (continuous line) and the non-selective one (dotted line), which transmits all messages regardless of their importance value, until energy is used up.

- Exponential: For an exponential distribution, we have

$$
p(x)=\frac{1}{a} \exp \left(-\frac{x}{a}\right) u(x),
$$

and

$$
H(\mu)=a \exp \left(-\frac{\mu}{a}\right)
$$

so that

$$
\lambda(e)=\lambda\left(e-E_{R}\right)+a \exp \left(-\frac{\mu(e)}{a}\right) u\left(e-E_{T}-E_{R}\right) .
$$

The variation of $\mu$ for an exponential distribution with $a=1, E_{T}=4$ and $E_{R}=1$ is illustrated in Fig. 3. The more restrictive threshold, compared to that one shown in Fig. 2(a) for the uniform distribution, gives rise to a higher increase in the expected reward with regard to the non-selective forwarder.

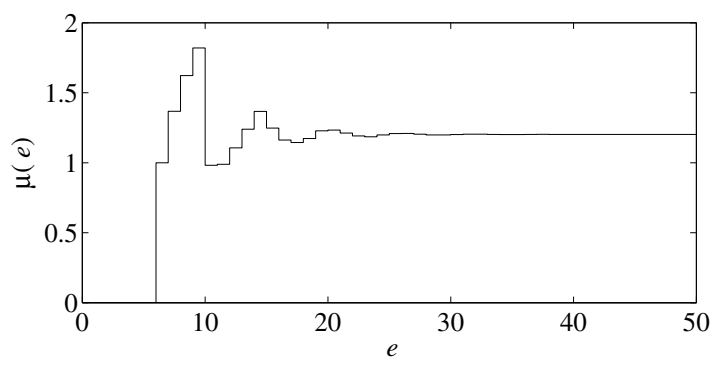

Fig. 3: Variation of the decision threshold with respect to the available energy. An exponential importance distribution with $a=1, E_{T}=4$ and $E_{R}=1$ is assumed.

- Pareto: For the Pareto-type distribution with PDF

$$
p(x)=\frac{a-1}{(1+x)^{a}} u(x),
$$

we have

$$
H(\mu)=\frac{1}{a-2} \frac{1}{(1+\mu)^{a-2}}
$$

so that

$$
\begin{aligned}
\lambda(e) & =\lambda\left(e-E_{R}\right) \\
& +\frac{1}{a-2} \frac{1}{(1+\mu(e))^{a-2}} u\left(e-E_{T}-E_{R}\right) .
\end{aligned}
$$

The evolution of $\mu$ for a Pareto distribution with $a=$ $3, E_{T}=4$ and $E_{R}=1$ is depicted in Fig. 4. Similar conclusions can be applied to this type of distribution.

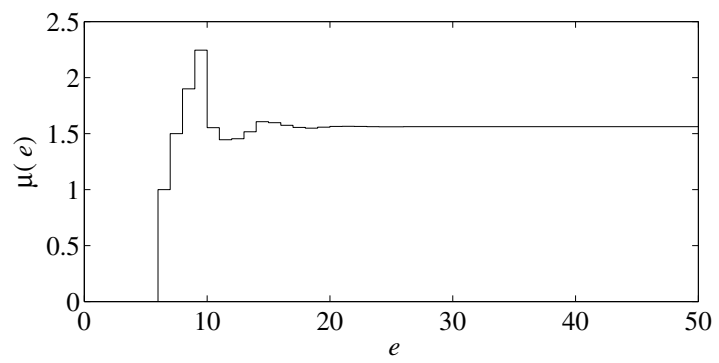

Fig. 4: Variation of the decision threshold with respect to the available energy. A Pareto importance distribution with $a=3, E_{T}=4$ and $E_{R}=1$ is assumed.

\section{ThE CONSTANT THRESHOLD TRANSMITTER}

For comparative purposes in the following sections, we will derive some expressions relative to selective transmission policies based on constant thresholds. Let us assume that node decisions are given by

$$
d_{k}=u\left(x_{k}-\mu_{c}\right) u\left(e_{k}-E_{1}\left(x_{k}\right)\right),
$$


where $\mu_{c}$ is a constant threshold. Note that if $\mu_{c}=0$ the constant-threshold transmitter reduces to the non-selective transmitter. Following an analysis similar to that exposed in Appendix A, we can write the expected reward of the constantthreshold transmitter as

$$
\begin{aligned}
\lambda_{k}(e)= & \mathbb{E}\left\{d_{k} x_{k} \mid e_{k}=e\right\}+\mathbb{E}\left\{\left(1-d_{k}\right) \lambda_{k+1}\left(e-E_{0}\left(x_{k}\right)\right)\right\} \\
& +\mathbb{E}\left\{d_{k} \lambda_{k+1}\left(e-E_{1}\left(x_{k}\right)\right)\right\} \\
= & \mathbb{E}\left\{u\left(x_{k}-\mu_{c}\right) u\left(e-E_{1}\left(x_{k}\right)\right) x_{k}\right\} \\
& +\left(1-\operatorname{Pr}\left\{x_{k} \geq \mu_{c}, e \geq E_{1}\left(x_{k}\right)\right\}\right) \\
& \cdot \mathbb{E}\left\{\lambda_{k+1}\left(e-E_{0}\left(x_{k}\right)\right) \mid\left(x_{k}<\mu_{c}\right) \text { OR }\left(e<E_{1}\left(x_{k}\right)\right)\right\} \\
& +\operatorname{Pr}\left\{x_{k} \geq \mu_{c}, e \geq E_{1}\left(x_{k}\right)\right\} \\
& \cdot \mathbb{E}\left\{\lambda_{k+1}\left(e-E_{1}\left(x_{k}\right)\right) \mid x_{k} \geq \mu_{c}, e \geq E_{1}\left(x_{k}\right)\right\} .
\end{aligned}
$$

In particular, for large $e$ (i.e. $e>\max _{x}\left\{E_{1}(x)\right\}$ ) and the stationary case

$$
\begin{aligned}
\lambda(e)= & \mathbb{E}\left\{u\left(x-\mu_{c}\right) x\right\} \\
& +\operatorname{Pr}\left\{x<\mu_{c}\right\} \mathbb{E}\left\{\lambda\left(e-E_{0}(x)\right) \mid x<\mu_{c}\right\} \\
& +\operatorname{Pr}\left\{x \geq \mu_{c}\right\} \mathbb{E}\left\{\lambda\left(e-E_{1}(x)\right) \mid x \geq \mu_{c}\right\} .
\end{aligned}
$$

Interestingly, for the constant profile case with $P_{I}=0$, $E_{1}(x)=E_{T}+E_{R}$ and $\mu_{c}=0, \lambda(e)$ can be computed explicitly using (12) as

$$
\begin{aligned}
\lambda_{k}(e) & =\sum_{i=k}^{\infty} \mathbb{E}\left\{u\left(e_{i}-E_{1}\left(x_{i}\right)\right) x_{i} \mid e_{k}=e\right\}= \\
& =\left\lfloor\frac{e}{E_{T}+E_{R}}\right\rfloor \mathbb{E}\{x\},
\end{aligned}
$$

where $\lfloor y\rfloor$ denotes the largest integer which is lower than $y$. So that (34) reflects the stepwise form shown in the example of Fig. 2.

\section{Asymptotic anALYSIS}

\subsection{Large energy threshold}

The above examples show that for large energy values $e$, the threshold converges to a constant value, and the expected reward tends to grow linearly. Both behaviors are closely related because, as (9) shows, the optimal threshold is the difference between two expected rewards. But this is also a general behavior of the constant-threshold transmitter. In this section, we discuss the asymptotic behavior of any selective transmitter in the stationary case. To do so, we first define the income rate of a selective transmitter.

Definition 1: The income rate of a selective transmitter with expected reward $\lambda(e)$ is defined as

$$
r=\lim _{e \rightarrow \infty} \frac{\lambda(e)}{e} .
$$

We start with the income rate of the constant-threshold transmitter, providing a formula and a proof for bounded energy profiles.

Theorem 3: Consider the selective transmitter given by (31), constant threshold $\mu_{c}$, and energy profiles with upper bound,
$B$, such that $E_{0}(x) \leq B$, and $E_{1}(x) \leq B$, for all $x$. Then, the income rate is given by

$$
r_{\mu_{c}}=\frac{\mathbb{E}\left\{u\left(x-\mu_{c}\right) x\right\}}{\left(1-P_{\mu_{c}}\right) \mathbb{E}\left\{E_{0}(x) \mid x<\mu_{c}\right\}+P_{\mu_{c}} \mathbb{E}\left\{E_{1}(x) \mid x \geq \mu_{c}\right\}}
$$

where $P_{\mu_{c}}=\operatorname{Pr}\left\{x \geq \mu_{c}\right\}$.

Proof: See Appendix C.

As a reference for comparison, we will consider the particular case of the non-selective transmitter, the particular case of the constant-threshold transmitter with $\mu_{c}=0$, in such a way that (36) reduces to

$$
r_{0}=\frac{\mathbb{E}\{x\}}{\mathbb{E}\left\{E_{1}(x)\right\}} .
$$

The following theorem provides a way to compute the income rate of the optimal selective transmission policy.

Theorem 4: The only threshold function $\mu(e, x)$ which is a solution of (14) and (15) and is constant with $e$ is given by

$$
\mu(e, x)=\mu(x)=\left(E_{1}(x)-E_{0}(x)\right) r,
$$

where $r$ is a solution of

$$
\mathbb{E}\left\{E_{0}(x)\right\} r=\mathbb{E}\left\{\left(x-\left(E_{1}(x)-E_{0}(x)\right) r\right)^{+}\right\} .
$$

Moreover, if $E_{1}(x) \geq E_{0}(x)$, for all $x$, this solution is unique.

Proof: See Appendix D.

An important consequence of Theorem 4 is that, if $\lim _{e \rightarrow \infty} \mu(e, x)$ exists, it must be equal to (38). Even though we will not show any theoretical convergence result, we have found a systematic empirical convergence, and we guess that this could be a general result for any importance distribution, provided it is stationary.

For the constant profile case, the asymptotic threshold (38) becomes

$$
\mu(x)=E_{T} r I_{x>0} .
$$

The recursive expression in (39) can be written as a function of $\mu^{*}=E_{T} r$ as

$$
\left(P_{I} E_{I}+\left(1-P_{I}\right) E_{R}\right) \mu^{*}=\left(1-P_{I}\right) E_{T} H\left(\mu^{*}\right)
$$

where $H\left(\mu^{*}\right)$ is given by (19). Defining

$$
\rho=\frac{\left(1-P_{I}\right) E_{T}}{P_{I} E_{I}+\left(1-P_{I}\right) E_{R}}
$$

we get

$$
\mu^{*}=\rho H\left(\mu^{*}\right) .
$$

\subsection{Gain of a selective forwarding scheme}

In this section we analyze asymptotically the advantages of the optimal selective scheme with regard to the non-selective one. To do so, we define the gain of a selective transmitter as the ratio of its income rate, $r$, and that of the non-selective transmitter, $r_{0}$,

$$
G=\frac{r}{r_{0}} .
$$

For the optimal selective transmitter in the constant profile case, combining (39) and (37), we get 


$$
\begin{aligned}
G & =\frac{\mu^{*} \mathbb{E}\left\{E_{1}(x)\right\}}{E_{T} \mathbb{E}\{x\}}=\frac{\mu^{*}\left(P_{I} E_{I}+\left(1-P_{I}\right)\left(E_{T}+E_{R}\right)\right)}{E_{T} \mathbb{E}\{x\}} \\
& =\left(1-P_{I}\right)\left(1+\rho^{-1}\right) \frac{\mu^{*}}{\mathbb{E}\{x\}} \\
& =\frac{1+\rho}{\rho} \frac{\mu^{*}}{\mathbb{E}\{x \mid x>0\}} .
\end{aligned}
$$

In the following, we compute the gain for several importance distributions.

\subsection{Examples}

Let us illustrate some examples taken from the constant profile case,

- Uniform Distribution: Substituting (23) into (43), we get

$$
\mu^{*}=\frac{1}{4} \rho\left(2-\mu^{*}\right)^{2},
$$

which can be solved for $\mu^{*}$ as

$$
\mu^{*}=2\left(\frac{1+\rho}{\rho}-\sqrt{\left(\frac{1+\rho}{\rho}\right)^{2}-1}\right)
$$

(the second root is higher than 2, which is not an admissible solution). Note that, for $\rho=4$, we get $\mu^{*}=1$, which agrees with the observation in Fig.2(a).

Therefore, the gain is given by

$$
G=2 \frac{1+\rho}{\rho}\left(\frac{1+\rho}{\rho}-\sqrt{\left(\frac{1+\rho}{\rho}\right)^{2}-1}\right) .
$$

- Exponential: Using (26) we find that $\mu^{*}$ is the solution of

$$
\mu^{*}=a W(\rho),
$$

where $W(x)=y$ is the real-valued Lambert's $W$ function which solves the equation $y e^{y}=x$ for $-1 \leq y \leq 0$ and $-1 / e \leq x \leq 0$ [22]. Thus,

$$
G=\left(1+\rho^{-1}\right) W(\rho) .
$$

Fig. 5 compares the gain of the uniform and the exponential distributions as a function of $\rho$. The graphic remarks that, under exponential distributions, the difference between the selective and the non-selective forwarding scheme is much more significant. The better performance of the exponential distribution compared to the uniform may be attributed to the tailed shape. We may think that, for a long-tailed distribution, the selective transmitter may be highly selective, saving energy for rare but extremely important messages. This intuition is corroborated by the following example.

- Pareto (one-sided): For this distribution, (29) can be used to conclude that $\mu^{*}$ is the solution of

$$
\mu^{*}=\frac{\rho}{(a-2)\left(1+\mu^{*}\right)^{a-2}} .
$$

Although for a generic $a$ this equation does not have an analytical solution (closed-form solutions for specific

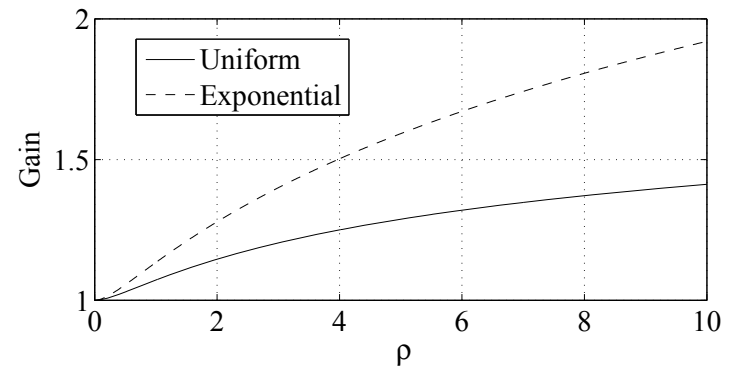

Fig. 5: Gain of the uniform and exponential distributions, as a function of $\rho$.

values of $a$ are possible), it can always be solved numerically. Fig. 6 shows the gain of the optimal selective forwarding policy under a Pareto distribution, for different values of parameter $a$. As stated before, it is corroborated that the gain achieves higher values for a Pareto distribution regarding to the other two distribution types. Besides, for the Pareto distribution, the higher the distribution parameter $a$ is, the lower the gain is.

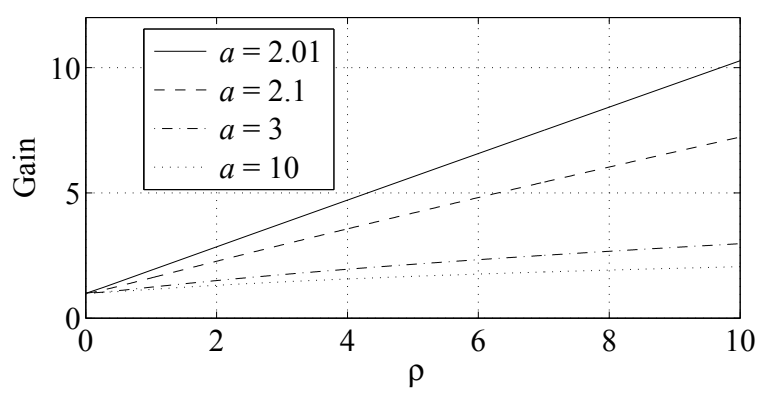

Fig. 6: Gain of the optimal selective forwarding policy under a Pareto distribution, for different values of parameter $a$. For values higher than 10 , the gain is approximately equal to the case $a=10$.

\subsection{Bounding the gain of a selective transmitter}

We can bound the gain of the optimal selective transmitter on a constant profile scenario by noting that, for any $\mu^{*} \geq 0$ and any importance distribution

$$
\begin{aligned}
H\left(\mu^{*}\right) & =\mathbb{E}\left\{\left(x-\mu^{*}\right) u\left(x-\mu^{*}\right) \mid x>0\right\} \\
& \leq \mathbb{E}\left\{x u\left(x-\mu^{*}\right) \mid x>0\right\} \leq \mathbb{E}\left\{x \frac{x}{\mu^{*}} \mid x>0\right\} \\
& =\frac{\mathbb{E}\left\{x^{2} \mid x>0\right\}}{\mu^{*}} .
\end{aligned}
$$

Using (43), we get

$$
\mu^{*} \leq \sqrt{\rho \mathbb{E}\left\{x^{2} \mid x>0\right\}} .
$$

Thus, the gain in (44) can be bounded as

$$
G \leq \frac{\sqrt{\mathbb{E}\left\{x^{2} \mid x>0\right\}}}{\mathbb{E}\{x \mid x>0\}} \frac{1+\rho}{\sqrt{\rho}} .
$$

Fig. 7 compares the normalized gain given by

$$
\bar{G}=\left(\frac{\sqrt{\mathbb{E}\left\{x^{2} \mid x>0\right\}}}{\mathbb{E}\{x \mid x>0\}}\right)^{-1} G
$$


with the theoretical bound, for different distribution types, in a $\log -\log$ scale. Note that, for large values of $\rho$, the bound is quite tight to the Pareto distribution.

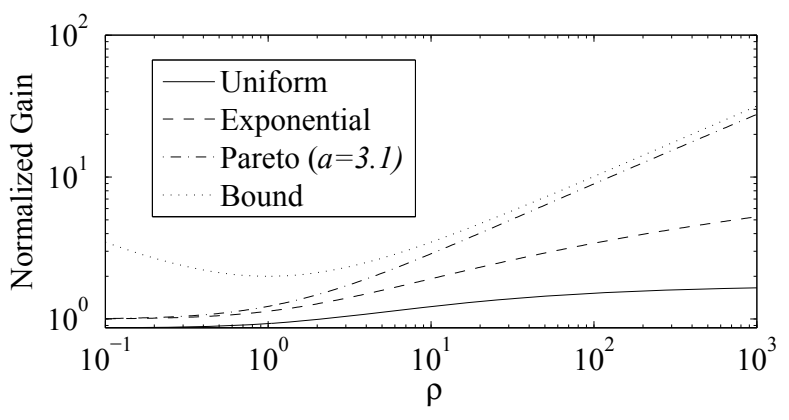

Fig. 7: Comparison of the normalized gain with the theoretical bound, for different importance distributions.

\subsection{Influence of idle times}

The above examples show that the gain of the optimal selective transmitter increases with $\rho$. By noting that $\rho$ in (42) is a decreasing function of $P_{I}$ and $E_{I}$, the influence of idle times becomes clear: as soon as the frequency of idle times or the idle energy expenses increases, the gain of the selective transmission scheme reduces. This effect will be observed in the experiments.

\section{Estimating Importance Distributions}

To obtain the optimum forwarding threshold the importance distribution of messages is required. However, in many practical scenarios $p\left(x_{k}\right)$ is either unknown or may change along time. To bypass this problem, $p\left(x_{k}\right)$ can be estimated in real time based on the available data $\left\{x_{\ell}, \ell=0, \ldots, k\right\}$ at time $k$.

We consider an approach based on a parametric estimation of $p\left(x_{k}\right)$ given by a Gamma distribution

$$
p(x \mid v, \theta)=x^{v-1} \frac{e^{-x / \theta}}{\theta^{v} \Gamma(v)}, \quad x, v, \theta>0 .
$$

The main reason for selecting (56) is that it does not require to store all importance values at each time and so, the estimation is not too much computationally expensive. Thresholds can be computed analytically by using the upper and lower incomplete gamma distribution $(\Gamma(a, x)$ and $\gamma(a, x)$, respectively),

$$
\begin{aligned}
& \mathbb{E}\{(x-\mu) u(x-\mu)\}=\frac{1}{\Gamma(v)}(\theta(\Gamma(v+1)-\gamma(v+1, \mu / \theta)) \\
& \quad-\mu(\Gamma(v)-\gamma(v, \mu / \theta))) .
\end{aligned}
$$

Let's define $\hat{q}_{k}=\frac{1}{k+1} \sum_{\ell=0}^{k} x_{\ell}$ and $\hat{t}_{k}=\frac{1}{k+1} \sum_{\ell=0}^{k} \ln \left(x_{\ell}\right)$. While the maximum-likelihood (ML) estimate $\hat{\theta}_{k}$ (ML estimate of $\theta$ at time $k$ ) is calculated as

$$
\hat{\theta}_{k}=\frac{\hat{q}_{k}}{\hat{v}_{k}},
$$

the ML estimate of parameter $v$ at time $k$, denoted by $\hat{v}_{k}$, can be obtained as the solution of $\ln \left(\hat{v}_{k}\right)-\psi\left(\hat{v}_{k}\right)=\ln \left(\hat{q}_{k}\right)-$ $\hat{t}_{k}$, where $\psi\left(\hat{v}_{k}\right)=\Gamma^{\prime}\left(\hat{v}_{k}\right) / \Gamma\left(\hat{v}_{k}\right)$ is the digamma function. Although there is not a closed-form solution for $\hat{v}_{k}$, it can be approximated as [23]

$$
\hat{v}_{k} \approx \frac{3-z_{k}+\sqrt{\left(z_{k}-3\right)^{2}+24 z_{k}}}{12 z_{k}},
$$

where $z_{k}=\ln \left(\hat{q}_{k}\right)-\hat{t}_{k}$. If accuracy were critical, closer approximations would be obtained iterating (see [24] for further details).

Note that $\hat{q}_{k}$ and $\hat{t}_{k}$ can be computed accumulatively, so that the importance sequence $x_{l}$ is not required to be stored (saving memory resources). Also, note that both sums in these equations can be exponentially weighted so as to cope with non-stationary importance distributions, as

$$
\begin{gathered}
\hat{q}_{k}=\frac{1-\alpha^{k+1}}{1-\alpha} \sum_{\ell=0}^{k} \alpha^{k-\ell} x_{\ell} \\
\hat{t}_{k}=\frac{1-\alpha^{k+1}}{1-\alpha} \sum_{\ell=0}^{k} \alpha^{k-\ell} \ln \left(x_{\ell}\right) .
\end{gathered}
$$

The design of optimal/efficient algorithms to estimate the importance distributions and calculate the optimal decision threshold is a complex problem that has to be thoroughly addressed. The scheme proposed in this section is a simple implementation that besides achieving good performance, can be used to gauge the influence of using estimates instead of the true statistics. Another alternative, considerably less expensive computationally speaking and in which we are working at present, consists of estimating the optimal threshold function by its asymptotic limit, which is also computed in real time based on the received data.

\section{EXPERIMENTS AND RESULTS}

In this section we test our novel selective message forwarding schemes in two different scenarios. All simulations have been conducted using Matlab.

\subsection{Isolated node}

Scenario A simulates an isolated energy-limited node. At each time $k$, the node receives a message of importance $x_{k}$ randomly generated according to a distribution $p(x)$. This distribution is known and independent of $k$ (stationary case). Next, a decision about transmitting the message is made. Three importance distributions have been considered: uniform, exponential, and Pareto. Distribution parameter $a$ is set to 1.8 and 3.5 for exponential and Pareto distributions, respectively. Samples belonging to the uniform distribution are generated according to $U(0,10)$. Recall, $x_{k}=0$ represents a silent time.

Performance of four different types of sensors is compared.

- Type NS (Non-Selective): Non-selective node. The threshold is set to $\mu=0$, so that it forwards all messages.

- Type OT (Optimal Transmitter): Optimal selective node. Threshold $\mu$ is computed according to (17) and (20), where nodes know the importance distribution $p(x)$.

- Type CT (Constant Threshold): Asymptotically optimal selective node. The sensor node establishes a constant 
threshold which is set to the asymptotic value of the optimal threshold given by (43).

- Type AT (Adaptive Transmitter): Adaptive selective node. The threshold is also computed following (17) and (20). Nevertheless, the node is unaware of $p(x)$ and it uses the estimation strategy exposed in Section 6 to know it, so that $\mu$ is computed according to (57).

The sensor's initial battery is set to $E=2000$ units. Energy expenses are also set to $E_{T}=4, E_{R}=1$ and $E_{I}=0$ units. A simulation finishes when the node runs out of battery. Results are averaged over 50 simulation runs.

Performance is assessed in terms of the importance sum of all transmitted messages, the mean value of the transmitted importances and the total number of transmitted messages. Results are summarized in Tables 1, 2 and 3.

The first observation is that the non-selective node transmits more messages than any type of the selective transmitters: approximately a $17 \%$ more in the uniform distribution, $32 \%$ in the exponential distribution and $47 \%$ in the Pareto distribution. Bearing in mind that the $N S$ sensor forwards all the messages it receives, it is hardly surprising that the number of transmitted messages is the same no matter the importance distribution (as opposed to selective transmitters). Nevertheless, on the one hand, the importance sum of all transmitted messages in a $N S$ sensor is the lowest in comparison with the selective transmitters. On the other hand, the mean value of the transmitted messages is lower for the $N S$ transmitter: nearly 7.5 for the selective transmitters with a uniform importance distribution against 5 in the $N S$ node; nearly 4 as opposed to 1.8 in an exponential importance distribution and 2.25 against 0.7 in a Pareto importance distribution. The mean value of the exponential and Pareto importance distributions are conditioned by the parameter selection, since it influences on the threshold, as it was shown in Section 5.3. Therefore, the simulated results confirm the less efficient behavior of the $N S$ transmitter.

TABLE 1: Averaged performance when the importance values are generated according to a uniform distribution - Scenario A

\begin{tabular}{|c|c|c|c|}
\hline \hline & $\begin{array}{c}\text { Avg. Total Imp. Tx } \\
\pm \text { std. deviation }\end{array}$ & Importance & Total Transmitted \\
& mean value & messages \\
\hline \hline Type NS & $1988.22 \pm 53.17$ & 4.97 & 400 \\
\hline Type OT & $2486.03 \pm 35.98$ & 7.48 & 332.50 \\
\hline Type CT & $2485.22 \pm 35.84$ & 7.48 & 332.22 \\
\hline Type AT & $2480.40 \pm 37.87$ & 7.59 & 326.82 \\
\hline \hline
\end{tabular}

TABLE 2: Averaged performance when the importance values are generated according to an exponential distribution- Scenario A

\begin{tabular}{|c|c|c|c|}
\hline \hline & $\begin{array}{c}\text { Avg. Total Imp. Tx } \\
\pm \text { std. deviation }\end{array}$ & $\begin{array}{c}\text { Importance } \\
\text { mean value }\end{array}$ & $\begin{array}{c}\text { Total Transmitted } \\
\text { messages }\end{array}$ \\
\hline \hline Type NS & $719.47 \pm 34.25$ & 1.80 & 400 \\
\hline Type OT & $1087.15 \pm 43.70$ & 3.98 & 273.46 \\
\hline Type CT & $1086.85 \pm 43.91$ & 3.98 & 272.92 \\
\hline Type AT & $1084.39 \pm 42.21$ & 3.94 & 275.34 \\
\hline \hline
\end{tabular}

Focusing on the selective transmitters, we observe that
$O T / C T$ nodes outperform the $A T$ node. Clearly, estimation errors penalize the performance of the $A T$ node compared to the optimum. In spite of it, its performance is close to the one achieved by the $O T$ sensor. Furthermore, it always yields a better result than the $N S$ transmitter. Regarding to the $C T$ node, results are similar to those of the $O T$ node (differences only appear when the node is close to use up its batteries). Performance clearly depends on the importance values of those messages arrived at the node when the battery level is scarce.

TABLE 3: Averaged performance when the importance values are generated according to a Pareto distribution - Scenario A

\begin{tabular}{|c|c|c|c|}
\hline \hline & $\begin{array}{c}\text { Avg. Total Imp. Tx } \\
\pm \text { std. deviation }\end{array}$ & $\begin{array}{c}\text { Importance } \\
\text { mean value }\end{array}$ & $\begin{array}{c}\text { Total Transmitted } \\
\text { messages }\end{array}$ \\
\hline \hline Type NS & $262.94 \pm 24.20$ & 0.66 & 400 \\
\hline Type OT & $473.47 \pm 38.35$ & 2.23 & 212.20 \\
\hline Type CT & $473.40 \pm 38.23$ & 2.23 & 211.82 \\
\hline Type AT & $469.06 \pm 40.79$ & 2.05 & 230.30 \\
\hline \hline
\end{tabular}

With the aim of obtaining a better comparison between these two selective sensors (OT and CT), their behavior under low battery resources is studied. In this case, the battery level is limited to 13 units, so that the maximum number of possible transmissions is two. Fig. 8 illustrates that, when batteries are scarce and the importance of the messages arriving at the sensor is low, the $O T$ sensor slightly outperforms the $C T$ sensor. This is because for small energy values, the optimum transmitter is more sensitive to energy changes.

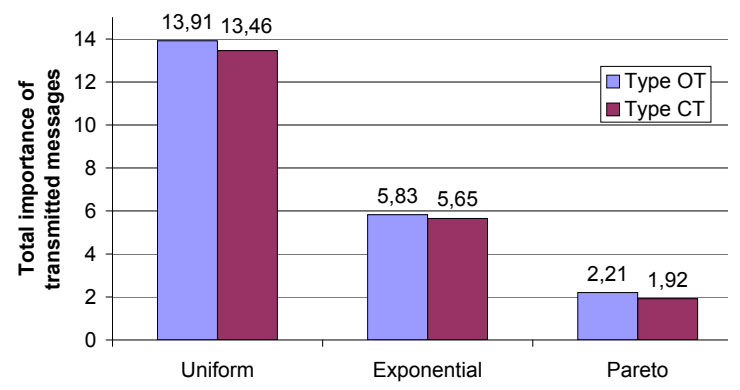

Fig. 8: Comparison between the optimal selective forwarder and the asymptotically optimal selective forwarder with low battery level.

Threshold variations as a function of the remaining energy $e$ under the influence of different frequencies of idle time $\left(P_{I}\right)$ are depicted in Fig. 9 for an $A T$ sensor.

Reception and idle energy expenses are fixed to the same amount ( $E_{R}=E_{I}=1$ units) and the node is initially provided with $E=2000$ units. The message importance follows a uniform distribution $U(0,10)$. As it can be observed, the more frequent the idle times increases, the lower the decision threshold is. The node is less selective when the opportunities to send true messages decreases, corroborating the theoretical results presented in this paper. Moreover, for high values of $e$ we observe strong oscillations in $\mu$. This oscillation occurs because during the first time instants the $A T$ node does not have enough samples to properly estimate the importance distribution. As the number of received messages increases, 


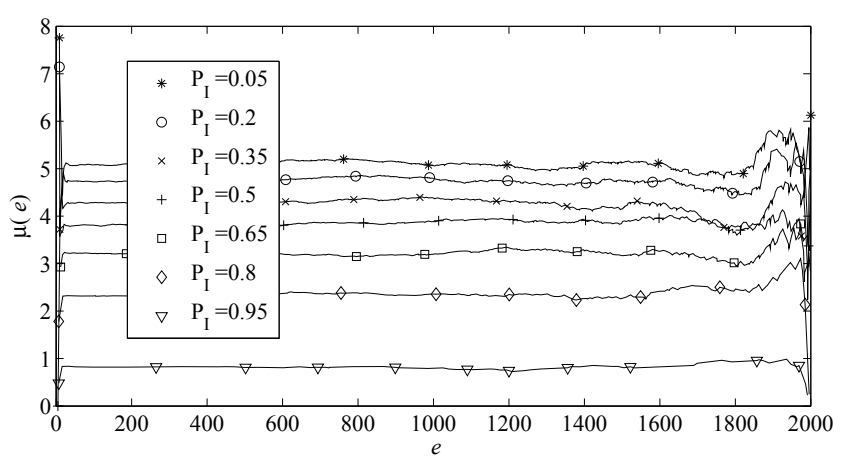

Fig. 9: Threshold evolution regarding to the remaining battery level $e$ in an isolated $A T$ sensor node for a uniform importance distribution $U(0,10)$. Different frequencies of being in idle state have been considered in a single run.

nearly constant thresholds are obtained and changes only appear when the node's battery is close to use up. The duration of the transitory phase will depend on the specific application. For instance, monitoring activities will not entail a long transitory phase. The reason is that during most of the time sensors are reporting a high number of consistent measurement values (the network is typically dense and the environment usually remains unchanged). On the other hand, in applications where nodes are not always collaborative, more time is needed to reach the long-term behavior of the network. A transitory phase might also appear if the importance distribution varies too drastically, since nodes would need some time to learn the new distribution (smooth changes should be easily tracked by the learning algorithm). Regardless of its duration, the impact of the transitory phase on the overall network performance is not necessarily critical, since high importance messages will be always transmitted.

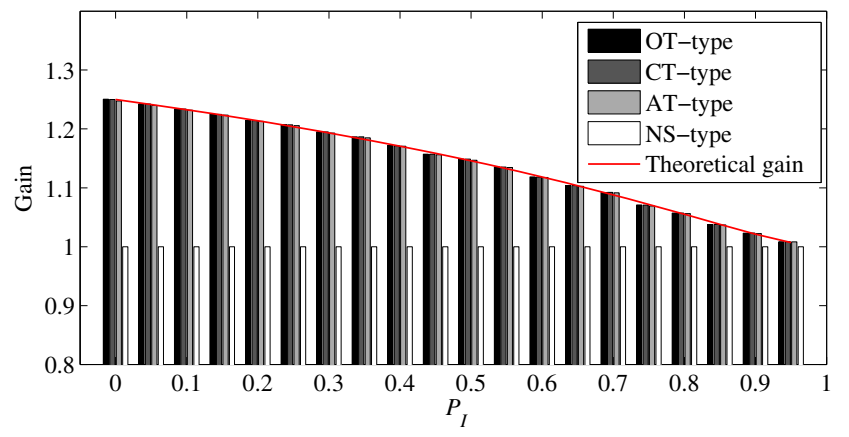

Fig. 10: Gain of the selective forwarding policy under a uniform importance distribution $U(0,10)$ for different values of $P_{I}$.

Fig. 10 shows the gain of the selective transmitters regarding to the non-selective forwarder for a uniform importance distribution and for different frequencies of idle time. As mentioned in Section 5.5, the gain of the selective forwarding scheme decreases as $P_{I}$ increases. Intuitively, it is easy to see that as $P_{I}$ approaches one (i.e., the node is in idle mode most of the time), the selective transmitter converges to the $N S$ transmitter. The same behavior is appreciated in the exponential and the Pareto importance distributions. In these cases, the gain is even higher than in the uniform case, which corroborates the theoretical study.

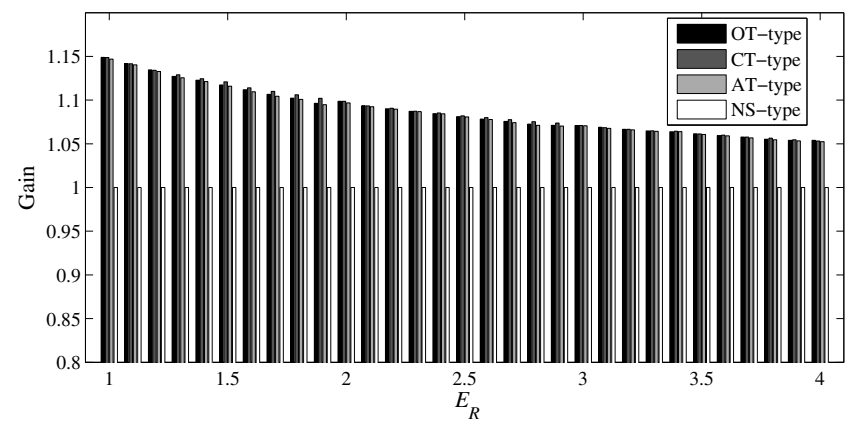

Fig. 11: Gain of the selective forwarding policy under a uniform importance distribution $U(0,10)$ for different values of $E_{R}$ and $P_{I}=$ 0.5 .

Finally, we have also investigated the behavior of the performance of the various forwarding strategies when a wide variety of energy models is considered. The study will help us to explore how our findings are influenced by the relative values of the energy consumptions. First of all, the gain of the aforementioned forwarding strategies for variable $E_{R}$ values is analyzed. Besides considering a uniform importance distribution, the transmission expense is set to $E_{T}=4$, the idle expense to $E_{I}=1$ and $P_{I}=0.5$. Fig. 11 shows a decreasing behavior in the gain for all type of selective transmitters when the $E_{R}$ value approaches to $E_{T}$. This behavior is expected since $\rho$ in (42) is a decreasing function of $E_{R}$. On the other hand, the $N S$ transmitter is not affected by the $E_{R}$ value since it will transmit the incoming messages in any case.

Fig. 12 focuses on the $O T$ transmitter in order to examine the behavior of the gain under the influence of the frequency of idle times and for different values of $E_{R}$. As it can be expected, as the frequency of idle times and the reception energy expenses increases, the gain of the selective transmission scheme reduces, according to (42).

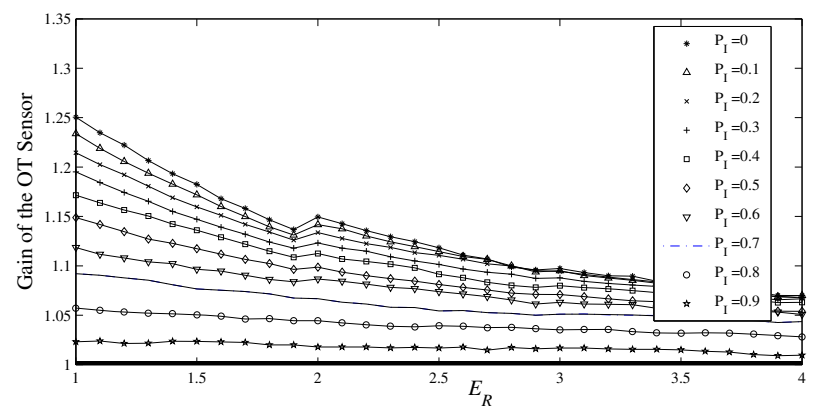

Fig. 12: Gain of the OT transmitter under a uniform importance distribution $U(0,10)$ for different values of $E_{R}$ and $P_{I}$.

\subsection{Sensor network}

The assumption of an isolated node is quite simple, so in scenario B, a sensor network is simulated. The sensor network is considered as a square area of $10 \times 10$, where 100 nodes 
have been uniformly random deployed. All nodes are identical and have the same initial resources (initial energy $E=200$ units) except for the sink, which is powerful in terms of energy resources. This static unique sink is always positioned at the right extreme of the field. $E_{T}, E_{R}$ and $E_{I}$ are set as in Section 7.1 when $P_{I}=0$. Sources are selected at random and keep transmitting messages of importances $x$ to the sink until network lifetime expires. Network lifetime is defined as the number of time slots achieved before the sink is isolated from its neighboring nodes. In order to simulate a more realistic set up, $x_{k} \in[0,10]$ for the three distribution types (a uniform, exponential and Pareto importance distributions) by adjusting their own parameters, with $x_{k}=0$ representing a silent time. Regarding to the forwarding schemes implemented, the same types of sensors that those from Section 7.1 are considered.

Nodes are considered as neighbors if they are placed within the transmission radius, which for simplicity reasons and due to power limitations is assumed to be the same for all nodes (i.e., a Unit Disk Graph model is assumed). Since nodes can only transmit messages inside their coverage area, they have geographical information about their own position, the location of their neighbors and the sink coordinates. Periodical "keep alive" beacons are sent to keep nodes updated. Link losses have also been included and so, the algorithm is made more robust by establishing a maximum number of retransmissions before discarding the message, which has been set to 5 in our simulations. It is naturally assumed that coverage areas are reciprocal, which is common when having a single omnidirectional antenna. Under this assumption nodes can listen to the channel and detect retransmissions of neighboring nodes before retransmitting the message again or discard it.

The routing algorithm implemented by the network follows a greedy forwarding scheme [25]. Although the disadvantages of the greedy forwarding algorithm are well-known (e.g. when the number of nodes close to the sink is small or there is a void), we choose this algorithm due to its simplicity, which will contribute to minimize its influence on the final results. This way, we can gauge better the effect of implementing our optimal selective schemes in a network, which indeed is the main objective of the simulations. It is worthy re-stressing that we are not proposing a new routing algorithm but a forwarding scheme with a selective mechanism and therefore, this scheme can also be integrated into other more efficient routing algorithms.

Performance is assessed in terms of the importance sum of all messages received by the sink, the mean value of these received importances, the number of transmissions made by origin nodes and the network lifetime (measured in time slots).

Experimental results are averaged over 50 different topologies which contain different samples of the three previous importance distributions.

Simulation results for scenario B are summarized in Tables 4, 5 and 6 . The numerical results confirm the conclusions extracted in scenario A and validate our theoretical claims. Basically, it is shown that the selective forwarding model outperforms the non-selective scheme.

Regardless of the distribution tested, both the mean value of the importance of messages received by the sink and the net-
TABLE 4: Averaged performance when the importance values are generated according to a uniform distribution - Scenario B

\begin{tabular}{|c|c|c|c|c|}
\hline \hline & Total Import. & Importance & Number of & Network \\
& Received Sink & mean value & Transmissions & Lifetime \\
\hline \hline Type NS & 1021.92 & 5.06 & 688.56 & 7896.00 \\
\hline Type OT & 1388.40 & 7.49 & 677.38 & 8467.90 \\
\hline Type CT & 1384.26 & 7.49 & 656.92 & 8441.08 \\
\hline Type AT & 1377.22 & 7.80 & 720.78 & 8812.74 \\
\hline \hline
\end{tabular}

TABLE 5: Averaged performance when the importance values are generated according to an exponential distribution - Scenario B

\begin{tabular}{|c|c|c|c|c|}
\hline \hline & Total Import. & Importance & Number of & Network \\
& Received Sink & mean value & Transmissions & Lifetime \\
\hline \hline Type NS & 331.72 & 1.76 & 672.84 & 7798.02 \\
\hline Type OT & 610.96 & 3.84 & 613.30 & 8758.00 \\
\hline Type CT & 609.45 & 3.86 & 596.82 & 8713.88 \\
\hline Type AT & 594.92 & 4.18 & 685.98 & 9309.56 \\
\hline \hline
\end{tabular}

work lifetime are higher when our selective forwarding scheme is implemented. As predicted in Section 3.3, differences are more noticeable when importance values are samples of a Pareto distribution.

Among the selective policies, $O T$ nodes exhibit the best performance. Nevertheless, differences among $O T, C T$ and $A T$ are smaller than in scenario A. The underlying reason is that decisions made at neighboring nodes and path losses may alter the shape of the original importance distribution. Since $A T$ nodes estimate the importance distribution $p(x)$ based on real received data, they are able to correct this alteration. This is not the case of $O T$ and $C T$ nodes, which calculate $\mu$ based on the original distribution, without accounting for the alterations introduced by the network. On the other hand, the transitory phase of $A T$ nodes makes them be more selective at the beginning, so that high importance messages are mainly transmitted in the first time instants originating an increase in the average of the importance value of the received messages. Besides, considering the fact that each node applies its own selective transmission policy together with channel losses, the network lifetime is increased given that nodes closer to the sink delay the exhaustion of their batteries and so, origin nodes are able to send more messages. The drawback, however, is a decrease in the total importance received at the sink.

TABLE 6: Averaged performance when the importance values are generated according to a Pareto distribution - Scenario B

\begin{tabular}{|c|c|c|c|c|}
\hline \hline & Total Import. & Importance & Number of & Network \\
& Received Sink & mean value & Transmissions & Lifetime \\
\hline \hline Type NS & 230.09 & 1.17 & 684.56 & 7875.76 \\
\hline Type OT & 630.76 & 5.67 & 466.50 & 11491.56 \\
\hline Type CT & 628.11 & 5.75 & 442.52 & 11420.48 \\
\hline Type AT & 510.60 & 3.86 & 666.36 & 9718.26 \\
\hline \hline
\end{tabular}




\section{Conclusions and Future Work}

This paper has introduced an optimum selective forwarding policy in wireless sensor networks as an energy-efficient scheme for data transmission. Messages, which were assumed to be graded with an importance value and which could be eventually discarded, were transmitted by sensor nodes according to a forwarding policy, which considered consumption patterns, available energy resources in nodes, the importance of the current message and the statistical description of such importances.

The optimal selective transmitter was derived, leading to an expression for the optimal decision which turned out to compare the received importance and the forwarding threshold, whose optimum value varies with time. Under certain simplifying operating conditions, a constant forwarding threshold which did not change along time and entailed asymptotic optimality, was also developed and closed-form expressions were obtained. Moreover, the gain of the selective forwarding policy compared to a non-selective one was quantified and it was proved to have a strong dependence on energy expenses (transmission, reception and idle), the frequency of idle times and the statistical distribution of importances. Finally, for cases were the importance distribution of messages was unknown (or it varied with time), a blind algorithm that caught this distribution on-the-fly based on the the received messages was proposed.

This study has also motivated the application of the selective forwarding model to two different evaluation cases, an isolated node and a sensor network. In both cases performance was assessed for different importance distributions. Numerical results validated our analytical claims and corroborated that the novel selective forwarding scheme clearly outperforms the non-selective one, even when idle times are considered. Results also evidenced that the simplified developed designs obtained a performance close to the optimal forwarder.

Several activities are under way within our research group in order to enhance the above contributions. First of all, a selective transmission policy aimed at improving the global performance in terms of quantity and quality of messages successfully arrived to the sink should be designed. Therefore, future work also includes the study of selective transmission in a sensor network, based on not only energy requirements, but also taking into account neighboring information. A preliminary work can be found in [19]. Also, to show the utility of the new optimization approach, our selective model will be applied to a target tracking scenario, whose first preliminary results can be found in [20]. Secondly, we are working on getting a solution for some model limitations, such as the computational load of the threshold computation.

\section{APPENDIX A}

\section{Proof of Theorem 1}

Defining the cumulative importance at time $k$ as

$$
s_{k}=\sum_{i=0}^{k} d_{i} x_{i},
$$

the dynamics of cumulative importances and energy can be described by the pair of equations

$$
\begin{gathered}
s_{k}=s_{k-1}+d_{k} x_{k} \\
e_{k+1}=e_{k}-d_{k} E_{1}\left(x_{k}\right)-\left(1-d_{k}\right) E_{0}\left(x_{k}\right),
\end{gathered}
$$

and $d_{k}=g\left(e_{k}, x_{k}\right)$ with the constraint in (6).

Note that the accumulated importance can be expressed as

$$
s_{\infty}=\sum_{i=0}^{\infty} d_{i} x_{i}=s_{k-1}+\sum_{i=k}^{\infty} d_{i} x_{i} .
$$

Since, for any $k, \mathbb{E}\left\{s_{\infty}\right\}=\int E\left\{s_{\infty} \mid e_{k}, x_{k}\right\} d P\left(e_{k}, x_{k}\right)$, maximizing $\mathbb{E}\left\{s_{\infty}\right\}$ is equivalent to maximize, for each $k$, $E\left\{s_{\infty} \mid e_{k}, x_{k}\right\},{ }^{3}$ which can be expressed as

$\mathbb{E}\left\{s_{\infty} \mid e_{k}, x_{k}\right\}=\mathbb{E}\left\{s_{k-1} \mid e_{k}, x_{k}\right\}+d_{k} x_{k}+\sum_{i=k+1}^{\infty} \mathbb{E}\left\{d_{i} x_{i} \mid e_{k}, x_{k}\right\}$

Since $d_{k}$ is a deterministic function of $e_{k}$ and $x_{k}$, we can write for any $i>k$,

$$
\begin{aligned}
\mathbb{E}\{ & \left.d_{i} x_{i} \mid e_{k}, x_{k}\right\} \\
= & \left(1-d_{k}\right) E\left\{d_{i} x_{i} \mid e_{k+1}=e_{k}-E_{0}\left(x_{k}\right), e_{k}, x_{k}\right\} \\
& +d_{k} E\left\{d_{i} x_{i} \mid e_{k+1}=e_{k}-E_{1}\left(x_{k}\right), e_{k}, x_{k}\right\},
\end{aligned}
$$

thus, replacing (67) into (66)

$$
\begin{aligned}
& \mathbb{E}\left\{s_{\infty} \mid e_{k}, x_{k}\right\}=\mathbb{E}\left\{s_{k-1} \mid e_{k}, x_{k}\right\} \\
& \quad+\quad d_{k}\left(x_{k}+\sum_{i=k+1}^{\infty} \mathbb{E}\left\{d_{i} x_{i} \mid e_{k+1}=e_{k}-E_{1}\left(x_{k}\right), e_{k}, x_{k}\right\}\right) \\
& \quad+\quad\left(1-d_{k}\right) \sum_{i=k+1}^{\infty} \mathbb{E}\left\{d_{i} x_{i} \mid e_{k+1}=e_{k}-E_{0}\left(x_{k}\right), e_{k}, x_{k}\right\}
\end{aligned}
$$

Since (i) for $i>k$ both $d_{i}$ and $x_{i}$ are independent of $x_{k}$ (the importance sequence, $x_{k}$, is statistically independent) and (ii) $e_{k+1}$ is fixed; we can remove $x_{k}$ and $e_{k}$ in the conditional expectations. Thus, we can use the definition of $\lambda_{k+1}$ in (12), to rewrite (68) as

$$
\begin{aligned}
& \mathbb{E}\left\{s_{\infty} \mid e_{k}, x_{k}\right\}=\mathbb{E}\left\{s_{k-1} \mid e_{k}, x_{k}\right\}+\lambda_{k+1}\left(e_{k}-E_{0}\left(x_{k}\right)\right) \\
& \quad+\left(x_{k}-\left[\lambda_{k+1}\left(e_{k}-E_{0}\left(x_{k}\right)\right)-\lambda_{k+1}\left(e_{k}-E_{1}\left(x_{k}\right)\right)\right]\right) d_{k} .
\end{aligned}
$$

Since the two first terms are fixed and do not depend on $d_{k}$, focus has to be placed on the third term. Defining $\mu_{k}\left(e_{k}, x_{k}\right)$ as in (9), the third term in (69) can be written as

$$
\left(x_{k}-\mu_{k}\left(e_{k}, x_{k}\right)\right) d_{k} .
$$

Clearly, the decision rule given by $d_{k}=1$ as soon as $x_{k} \geq$ $\mu_{k}\left(e_{k}, x_{k}\right)$ (so as to maximize (70)) and $e_{k} \geq E_{1}\left(x_{k}\right)$ (so as to satisfy the constraint in (6)) and $d_{k}=0$ otherwise, is optimal in the sense of maximizing $\mathbb{E}\left\{s_{\infty} \mid e_{k}, x_{k}\right\}$.

3. An intuitive explanation for the equivalence between maximizing $E\left\{s_{\infty}\right\}$ and $E\left\{s_{\infty} \mid e_{k}, x_{k}\right\}$ is the following: no matter what a selective transmission scheme has done up to time $k-1$, the best that can be done at time $k$ is maximizing $E\left\{s_{\infty} \mid e_{k}, x_{k}\right\}$. If this rule is applied at every time $k$, the unconditional expectation, $E\left\{s_{\infty}\right\}$, is maximized. 
The recursive computation of $\lambda_{k}(e)$ in (10) is the only result that remains to be proved. To do so we note that, for any $i>k$,

$$
\begin{aligned}
\mathbb{E}\left\{d_{i} x_{i} \mid e_{k}\right\}=\operatorname{Pr}\left\{d_{k}=0 \mid e_{k}\right\} \mathbb{E}\left\{d_{i} x_{i} \mid e_{k}, d_{k}=0\right\} \\
\quad+\operatorname{Pr}\left\{d_{k}=1 \mid e_{k}\right\} \mathbb{E}\left\{d_{i} x_{i} \mid e_{k}, d_{k}=1\right\} \\
=\left(1-\operatorname{Pr}\left\{x_{k} \geq \mu_{k}\left(e_{k}, x_{k}\right), e_{k} \geq E_{1}\left(x_{k}\right) \mid e_{k}\right\}\right) \\
\quad \cdot \mathbb{E}\left\{\mathbb{E}\left\{d_{i} x_{i} \mid e_{k+1}=e_{k}-E_{0}\left(x_{k}\right), e_{k}, d_{k}=0\right\}\right\} \\
\quad+\operatorname{Pr}\left\{x_{k} \geq \mu_{k}\left(e_{k}, x_{k}\right), e_{k} \geq E_{1}\left(x_{k}\right) \mid e_{k}\right\} \\
\quad \cdot \mathbb{E}\left\{\mathbb{E}\left\{d_{i} x_{i} \mid e_{k+1}=e_{k}-E_{1}\left(x_{k}\right), e_{k}, d_{k}=1\right\}\right\},
\end{aligned}
$$

(where the external expectation must be taken over $\left.x_{k} \mid e_{k}, d_{k}=0\right)$. Using the definition of $\lambda_{k}(e)$ in (12) and capitalizing on (71), we find

$$
\begin{aligned}
\lambda_{k}(e) & =\sum_{i=k}^{\infty} \mathbb{E}\left\{d_{i} x_{i} \mid e_{k}=e\right\}=\mathbb{E}\left\{d_{k} x_{k} \mid e_{k}=e\right\} \\
& +\sum_{i=k+1}^{\infty} \mathbb{E}\left\{\mathbb{E}\left\{d_{i} x_{i} \mid e_{k}=e, x_{k}\right\}\right\}
\end{aligned}
$$

where the outer expectation applies over $x_{k}$. Taking into account that $d_{k}$ only depends on $e_{k}$ and $x_{k}$, the conditions in the inner expectation operators determine uniquely $d_{k}$ and, thus, we can write

$$
\begin{aligned}
& \lambda_{k}(e)= \mathbb{E}\left\{d_{k} x_{k} \mid e_{k}=e\right\} \\
&+\sum_{i=k+1}^{\infty} \mathbb{E}\left\{\left(1-d_{k}\right) \mathbb{E}\left\{d_{i} x_{i} \mid e_{k+1}=e-E_{0}\left(x_{k}\right), x_{k}\right\}\right. \\
&\left.\quad+d_{k} \mathbb{E}\left\{d_{i} x_{i} \mid e_{k+1}=e-E_{1}\left(x_{k}\right), x_{k}\right\}\right\} \\
&=\mathbb{E}\left\{d_{k} x_{k} \mid e_{k}=e\right\}+\mathbb{E}\left\{\left(1-d_{k}\right) \lambda_{k+1}\left(e-E_{0}\left(x_{k}\right)\right)\right\} \\
&+ \\
&+\mathbb{E}\left\{d_{k} \lambda_{k+1}\left(e-E_{1}\left(x_{k}\right)\right)\right\} \\
&=\mathbb{E}\left\{d_{k} x_{k} \mid e_{k}=e\right\}-\mathbb{E}\left\{d_{k} \mu_{k+1}\left(e, x_{k}\right)\right\} \\
& \quad+\mathbb{E}\left\{\lambda_{k+1}\left(e-E_{0}\left(x_{k}\right)\right)\right\} \\
&=\mathbb{E}\left\{d_{k}\left(x_{k}-\mu_{k}\left(e_{k}, x_{k}\right)\right) \mid e_{k}=e\right\} \\
&+\mathbb{E}\left\{\lambda_{k+1}\left(e-E_{0}\left(x_{k}\right)\right)\right\} \\
&= \mathbb{E}\left\{\lambda_{k+1}\left(e-E_{0}\left(x_{k}\right)\right)\right\} \\
&+\mathbb{E}\left\{\left(x_{k}-\mu_{k}\left(e, x_{k}\right)\right)+u\left(e-E_{1}\left(x_{k}\right)\right)\right\} .
\end{aligned}
$$

The initial value can be computed using (6): if there is no available energy, transmissions are not possible: mathematically, if $e_{k}=0, d_{i}=0$ for $i>k$, so that (12) becomes

$$
\lambda_{k}(0)=0, \quad \text { for any } k \text {. }
$$

Combining (73) and (74) we get (10), completing the proof.

\section{APPENDIX B}

\section{ProOF OF THEOREM 2}

Let's define the minimum energy consumed per time as $\epsilon=\min _{i, x}\left\{E_{i}(x)\right\}$. We prove the theorem by induction, by showing that $\lambda_{k}(e)$ does not depend on $k$ for $e \leq n \epsilon$, for any $n$. This is true for $n=0$, because $\lambda_{k}(0)=0$. Now, let us assume that $\lambda_{k}(e)$ does not depend on $k$ for $e \leq n \epsilon$. If $n \epsilon<e \leq(n+1) \epsilon$, by (9) we find that $\mu_{k}(e, x)$ does not depend on $k$. Thus, using (10), and taking into account that expectations are taken over $x_{k}$, whose distribution does not depend on $k$, we find that $\lambda_{k}(e)$ does not depend on $k$, which completes the proof.

\section{ApPENDIX C ProOF OF THEOREM 3}

Defining

$$
z(e)=\lambda(e)-r_{\mu_{c}} e \cdot u(e)
$$

where $r_{\mu_{c}}$ is given by (36), we have, for large $e$ (i.e., $e>B$ )

$$
\begin{aligned}
z(e)= & \mathbb{E}\left\{u\left(x-\mu_{c}\right) x\right\}-r_{\mu_{c}} e \\
& +\operatorname{Pr}\left\{x<\mu_{c}\right\} \mathbb{E}\left\{\lambda\left(e-E_{0}(x)\right) \mid x<\mu_{c}\right\} \\
& +\operatorname{Pr}\left\{x \geq \mu_{c}\right\} \mathbb{E}\left\{\lambda\left(e-E_{1}(x)\right) \mid x \geq \mu_{c}\right\} \\
= & \mathbb{E}\left\{u\left(x-\mu_{c}\right) x\right\}-r_{\mu_{c}} e \\
& +\operatorname{Pr}\left\{x<\mu_{c}\right\} \mathbb{E}\left\{z\left(e-E_{0}(x)\right) \mid x<\mu_{c}\right\} \\
& +\operatorname{Pr}\left\{x<\mu_{c}\right\} r_{\mu_{c}} \mathbb{E}\left\{e-E_{0}(x) \mid x<\mu_{c}\right\} \\
& +\operatorname{Pr}\left\{x \geq \mu_{c}\right\} \mathbb{E}\left\{z\left(e-E_{1}(x)\right) \mid x \geq \mu_{c}\right\} \\
& +\operatorname{Pr}\left\{x \geq \mu_{c}\right\} r_{\mu_{c}} \mathbb{E}\left\{e-E_{1}(x) \mid x \geq \mu_{c}\right\} \\
= & \mathbb{E}\left\{u\left(x-\mu_{c}\right) x\right\} \\
& +\operatorname{Pr}\left\{x<\mu_{c}\right\} \mathbb{E}\left\{z\left(e-E_{0}(x)\right) \mid x<\mu_{c}\right\} \\
& +\operatorname{Pr}\left\{x<\mu_{c}\right\} r_{\mu_{c}} \mathbb{E}\left\{E_{0}(x) \mid x<\mu_{c}\right\} \\
& +\operatorname{Pr}\left\{x \geq \mu_{c}\right\} \mathbb{E}\left\{z\left(e-E_{1}(x)\right) \mid x \geq \mu_{c}\right\} \\
& +\operatorname{Pr}\left\{x \geq \mu_{c}\right\} r_{\mu_{c}} \mathbb{E}\left\{E_{1}(x) \mid x \geq \mu_{c}\right\} \\
= & \operatorname{Pr}\left\{x<\mu_{c}\right\} \mathbb{E}\left\{z\left(e-E_{0}(x)\right) \mid x<\mu_{c}\right\} \\
& +\operatorname{Pr}\left\{x \geq \mu_{c}\right\} \mathbb{E}\left\{z\left(e-E_{1}(x)\right) \mid x \geq \mu_{c}\right\} .
\end{aligned}
$$

Defining the random variable

$$
\epsilon=E_{0}(x) I_{x<\mu_{c}}+E_{1}(x) I_{x \geq \mu_{c}}
$$

we have

$$
\begin{aligned}
z(e) & =\mathbb{E}\{z(e-\epsilon)\} \\
& =P\{\epsilon=0\} z(e)+P\{\epsilon>0\} \mathbb{E}\{z(e-\epsilon) \mid \epsilon>0\} .
\end{aligned}
$$

Thus,

$$
z(e)=\mathbb{E}\{z(e-\epsilon) \mid \epsilon>0\} .
$$

Since $z(e)$ is a weighted average of previous values, there should exist some values $\epsilon_{l}>0$ and $\epsilon_{u}>0$ such that

$$
z\left(e-\epsilon_{l}\right)<z(e)<z\left(e-\epsilon_{u}\right) .
$$

Applying this inequalities iteratively, we can prove by induction that

$$
z\left(e_{l}\right) \leq z(e) \leq z\left(e_{u}\right), \quad \text { for some } e_{l}, e_{u} \leq B
$$

But, since $\lambda$ is finite for finite $e, \lambda$ is bounded in $[0, B]$ so $z$ is also bounded in $[0, B]$. Thus, using (81) we conclude that $z(e)$ is bounded in $\mathbb{R}$. Therefore, we can compute the income rate as

$$
\lim _{e \rightarrow \infty} \frac{\lambda(e)}{e}=\lim _{e \rightarrow \infty} \frac{z(e)+r_{\mu_{c}} e}{e}=r_{\mu_{c}}
$$




\section{APPENDIX D ProOF OF THEOREM 4}

Let's assume that the threshold function does not depend on the energy level, so that $\mu(e, x)=\mu(x)$. Then, using (14), we can write

$$
\lambda(e)=\lambda(e-\Delta(x))+\mu(x)
$$

where

$$
\Delta(x)=E_{1}(x)-E_{0}(x) .
$$

Defining

$$
g_{x}(e)=\lambda(e)-\frac{\mu(x)}{\Delta(x)} e
$$

Eq. (83) implies that $g_{x}(e)$ is a periodic function with period $\Delta(x)$. But this is impossible if $\frac{\mu(x)}{\Delta(x)}$ varies with $x$ (because the difference between two periodic functions cannot be a (non constant) linear function. Thus,

$$
\mu(x) / \Delta(x)=r
$$

for some constant $r$, and

$$
g_{x}(e)=g(e)=\lambda(e)-r e .
$$

Combining (15), (83) and (87) we can write

$$
\begin{aligned}
g(e)+\frac{\mu(x)}{\Delta(x)} e= & \mathbb{E}\left\{g\left(e-E_{0}(x)\right)+r\left(e-E_{0}(x)\right)\right\} \\
& +\mathbb{E}\left\{(x-\mu(x))^{+}\right\} .
\end{aligned}
$$

Thus

$g(e)-\mathbb{E}\left\{g\left(e-E_{0}(x)\right)\right\}=-\mathbb{E}\left\{r E_{0}(x)\right\}+\mathbb{E}\left\{(x-\mu(x))^{+}\right\}$

Integrating the above equation with respect to $e$ over a full period and, noting that $\int_{\Delta(x)} g(e) d e=\int_{\Delta(x)} g\left(e-E_{0}(x)\right) d e$ we get

$$
r \mathbb{E}\left\{E_{0}(x)\right\}=\mathbb{E}\left\{(x-\Delta(x) r)^{+}\right\},
$$

which is equivalent to (39). To show that the solution of (90) is unique, note that the left-hand side is a strictly growing function of $r$ while the right-hand side is a non-increasing function, because

$$
\frac{d \mathbb{E}\left\{(x-\Delta(x) r)^{+}\right\}}{d r}=-\mathbb{E}\{\Delta(x) u(x-\Delta(x) r)\},
$$

which is always non positive. Since a strictly increasing function intersects with a non-increasing function in at most one single point, the solution is unique.

\section{ACKNOWLEDGMENT}

This work was partially funded by projects TEC2005-06766C03-02 and TEC2008-01348 from the Spanish Ministry of Education and Science and CAM-UC3M project CCG07UC3M/TIC-3380.

\section{REFERENCES}

[1] I. F. Akyildiz, W. Su, Y. Sankarasubramaniam, and E. Cayirci, "A Survey on Sensor Networks," IEEE Comm. Magazine, vol. 40, no. 8, pp. 102114, Aug. 2002.

[2] Q. Jiang and D. Manivannan, "Routing Protocols for Sensor Networks," in Proc. 1st IEEE Consumer Comm. and Networking Conf. (CCNC '04), Jan. 2004, pp. 93-98.

[3] S. B. Lee, K. Kwak, and A. Campbell, "Solicitation-based Forwarding for Sensor Networks," in Proc. 3th Annual IEEE Comm. Society Conf. on Sensor and Ad Hoc Comm. and Networks (SECON '06), Sept. 2006, pp. 90-99.

[4] G. Chelius, E. Fleury, and T. Mignon, "Lower and upper bounds for minimum energy broadcast and sensing problems in sensor networks," in Proc. 11th Int'l Conf. Parallel and Distributed Systems, vol. 2, July 2005, pp. 88-92.

[5] W. Ye, J. Heidemann, and D. Estrin, "An Energy-Efficient MAC Protocol for Wireless Sensor Networks," in Proc. 21st Annual Joint Conf. IEEE Computer and Comm. Societies (INFOCOM '02), vol. 3, 2002, pp. 1567 $-1576$.

[6] D. Dardari, A. Conti, C. Buratti, and R. Verdone, "Mathematical Evaluation of Environmental Monitoring Estimation Error through EnergyEfficient Wireless Sensor Networks," IEEE Transactions on Mobile Computing, vol. 6, no. 7, pp. 790-802, July 2007.

[7] A. D. Wood and J. A. Stankovic, "Denial of Service in Sensor Networks," IEEE Computer, vol. 35, no. 10, pp. 54-62, Oct. 2002.

[8] R. Muraleedharan, Y. Yan, and L. A. Osadciw, "Increased Efficiency of Face Recognition System Using Wireless Sensor Network," Journal of Cybernetics, Systemics, and Informatics, Nov. 2006.

[9] L. Wischhof, A. Ebner, H. Rohling, M.Lott, and R. Halfmann, "SOTIS A Self-Organizing Traffic Information System,' in Proc. 57th IEEE Semiannual Vehicular Technology Conf. (VTC '03), vol. 4, Apr. 2003 , pp. 2442- 2446 .

[10] M. Athanassoulis, I. Alagiannis, and S. Hadjiefthymiades, "Energy Efficiency in Wireless Sensor Networks: A Utility-Based Architecture," in Proc. 13th European Wireless Conf. (EW'07), Apr. 2007.

[11] V. Shnayder, B. Chen, K. Lorincz, T. Fulford-Jones, and M. Welsh, "Sensor Networks for Medical Care," in Proc. 3rd Int'l Conf. on Embedded networked sensor systems, Nov. 2005.

[12] J. Qiu, Y. Tao, and S. Lu, Grid and Cooperative Computing. Springer Berlin / Heidelberg, 2005, vol. 3795/2005, ch. Differentiated Application Independent Data Aggregation in Wireless Sensor Networks, pp. 529 534.

[13] G. Merrett, B. Al-Hashimi, N. White, and N. Harris, "Information Managed Wireless Sensor Networks with Energy Aware Nodes," in Proc. NSTI Nanotechnology Conf. and Trade Show (NanoTech '05), May 2005, pp. 367-370.

[14] S. J. Mujumdar, "Prioritized Geographical Routing in Sensor Networks," Master's thesis, Vanderbilt University, Tennessee, May 2004.

[15] J. Rivera, G. Bojorquez, M. Chacon, G. Herrera, and M. Carrillo, "A Fuzzy Message Priority Arbitration Approach for Sensor Networks," in Proc. North American Fuzzy Information Processing Society (NAFIPS '07), June 2007, pp. 586-591.

[16] R. Arroyo-Valles, A.G. Marqués, J. Vinagre-Díaz, and J. Cid-Sueiro, "A Bayesian Decision Model for Intelligent Routing in Sensor Networks," in Proc. 3rd IEEE Int'l Symp. on Wireless Comm. Systems (ISWCS '06), Sept. 2006.

[17] R. Arroyo-Valles, A.G. Marqués, and J. Cid-Sueiro, "Energy-aware Geographic Forwarding of Prioritized Messages in Wireless Sensor Networks," in Proc. 4th IEEE Int'l Conf. on Mobile Ad-hoc and Sensor Systems (MASS '07), Oct. 2007.

[18] R. Arroyo-Valles, R. Alaiz-Rodriguez, A. Guerrero-Curieses, and J. CidSueiro, "Q-Probabilistic Routing in Wireless Sensor Network," in Proc. 3th Int'l Conf. Intelligent Sensor, Sensor Networks and Information Processing (ISSNIP '07), Dec. 2007.

[19] R. Arroyo-Valles, A.G. Marqués, and J. Cid-Sueiro, "Energy-efficient Selective Forwarding for Sensor Networks," in Proc. Workshop on Energy in Wireless Sensor Networks (WEWSN'08), in conjunction with DCOSS'08, June 2008.

[20] R. Arroyo-Valles, S. Pino-Povedano, J. Cid-Sueiro, and F.J. GonzalezSerrano "Distributed Target Tracking with Selective Transmitters in energy-constrained Sensor Networks," in Proc. 4th Int. Conf. on Intelligent Sensors, Sensor Nertworks and Information Processing (ISSNIP'08), Dec. 2008.

[21] L. I. Sennott, Stochastic Dynamic Programming and the Control of Queueing Systems. Wiley-Interscience, 1999. 
[22] R. M. Corless, G. H. Gonnet, D. E. G. Hare, D. J. Jeffrey, and D. E. Knuth, "On the Lambert W function," Advances in Computational Mathematics, vol. 5, pp. 329-359, 1996.

[23] M. Maddah, W. W. III, S. Warfield, C. Westin, and W. Grimson, "Probabilistic Clustering and Quantitative Analysis of White Matter Fiber Tracts," in Proc. 20th Int'l Conf. on Information Processing in Medical Imaging (IPMI 2007), July 2007, pp. 372-383.

[24] S. Choi and R. Wette, "Maximum Likehood Estimation of the Parameters of the Gamma Distribution and Their Bias," Technometrics, vol. 2, no. 4, pp. 683-690, Nov. 1969.

[25] B. Karp and H. Kung, "Greedy Perimeter Stateless Routing for Wireless Networks," in Proc. 6th Annual ACM/IEEE Int'l Conf. on Mobile Computing and Networking (MobiCom 2000), Aug. 2000, pp. 243-254.

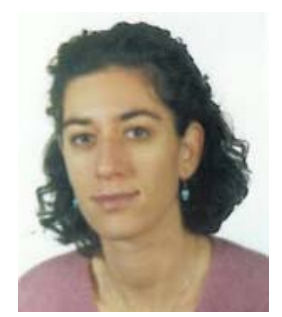

Rocío Arroyo-Valles received the M.S. degree in Telecommunications Engineering from the Universidad de Valladolid, Valladolid, Spain, in 2004. She is currently working towards a Ph.D. degree at the Universidad Carlos III de Madrid, Madrid, Spain.

Her current research focuses on intelligent routing and selective transmission in sensor networks, and multimedia signal processing.

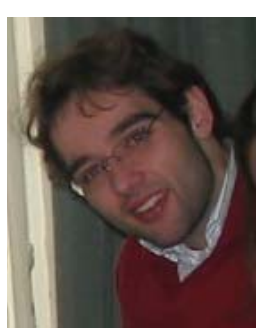

Antonio G. Marques (M'07) received the degree in Telecommunication Engineering and the $\mathrm{Ph}$. D. degree both with highest honors from the Universidad Carlos III de Madrid, Madrid, Spain, in 2002 and 2007 respectively. In 2003 he joined the Department of Signal Theory and Communications, Universidad Rey Juan Carlos, Madrid, Spain, where he currently develops his research and teaching activities as an Assistant Professor. Since 2005 he is also a Visiting Researcher at the Department of Electrical Engineering, University of Minnesota, MN, USA.

His research interests lie in the areas of communication theory, signal processing, and networking. His current research focuses on channel state information designs, energy-efficient resource allocation, and wireless ad hoc and sensor networks.

Dr. Marques work brought him several awards in distinctive international conferences including ICASSP 2007.

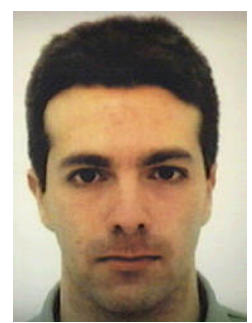

Jesús Cid-Sueiro (M95) received the degree in Telecommunication Engineering from Universidad de Vigo, Spain, in 1990 and the Ph.D. degree from Universidad Politecnica de Madrid, Spain, in 1994. Since 1999, he has been an Associate Professor in the Department of Signal Theory and Communications, Universidad Carlos III de Madrid, Spain.

His main research interests include computational intelligence, neural networks, Bayesian methods and their applications to sensor networks, communications and multimedia processing. 